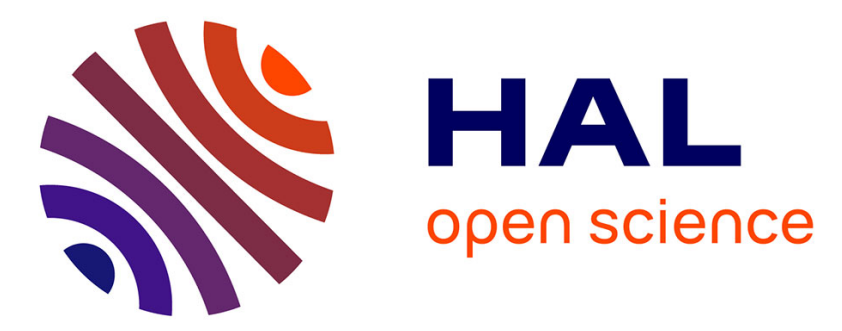

\title{
Spectral features of lightning-induced ion cyclotron waves at low latitudes: DEMETER observations and simulation
}

D. R. Shklyar, L.R.O. Storey, J Chum, F Jiřriček, F Němec, Michel Parrot, Ondřej Santolík, E. E. Titova

\section{To cite this version:}

D. R. Shklyar, L.R.O. Storey, J Chum, F Jiř́čcek, F Němec, et al.. Spectral features of lightning-induced ion cyclotron waves at low latitudes: DEMETER observations and simulation. Journal of Geophysical Research Space Physics, 2012, 117, A12206 (16 p.). 10.1029/2012JA018016 . insu-01179844

\section{HAL Id: insu-01179844 \\ https://hal-insu.archives-ouvertes.fr/insu-01179844}

Submitted on 23 Jul 2015

HAL is a multi-disciplinary open access archive for the deposit and dissemination of scientific research documents, whether they are published or not. The documents may come from teaching and research institutions in France or abroad, or from public or private research centers.
L'archive ouverte pluridisciplinaire HAL, est destinée au dépôt et à la diffusion de documents scientifiques de niveau recherche, publiés ou non, émanant des établissements d'enseignement et de recherche français ou étrangers, des laboratoires publics ou privés. 


\title{
Spectral features of lightning-induced ion cyclotron waves at low latitudes: DEMETER observations and simulation
}

\author{
D. R. Shklyar, ${ }^{1,2}$ L. R. O. Storey, ${ }^{3}$ J. Chum, ${ }^{4}$ F. Jiříček, ${ }^{4}$ F. Němec, ${ }^{5}$ M. Parrot, ${ }^{6}$ \\ O. Santolik, ${ }^{4,5}$ and E. E. Titova ${ }^{7}$ \\ Received 12 June 2012; revised 12 October 2012; accepted 15 October 2012; published 7 December 2012.
}

[1] We use a comprehensive analysis of 6-component ELF wave data from the DEMETER satellite to study proton whistlers, placing emphasis on low-latitude events originating from lightning strokes in the hemisphere opposite to the hemisphere of observation. In this case, the formation of proton whistlers does not involve mode conversion caused by a strong mode coupling at a crossover frequency, although a polarization reversal remains an important element in formation of the phenomenon. DEMETER measurements of the six electromagnetic field components in the frequency band below $1000 \mathrm{~Hz}$ make it possible to determine not only the dynamic spectrum, but also the wave polarization, the wave normal angle, and the normalized parallel component of the Poynting vector. This permits us to address fine features of proton whistlers, in particular, we show that the deviation of the upper cutoff frequency from the equatorial cyclotron frequency is related to the Doppler shift. Experimental study of proton whistlers is supplemented by an investigation of ion cyclotron wave propagation in a multicomponent magnetoplasma and by numerical modeling of spectrograms, both in the frame of geometrical optics.

Citation: Shklyar, D. R., L. R. O. Storey, J. Chum, F. Jiříček, F. Němec, M. Parrot, O. Santolik, and E. E. Titova (2012), Spectral features of lightning-induced ion cyclotron waves at low latitudes: DEMETER observations and simulation, J. Geophys. Res., 117, A12206, doi:10.1029/2012JA018016.

\section{Introduction}

[2] Lightning strokes in the Earth's atmosphere are the source of various wave phenomena observed onboard spacecraft. These phenomena (as well as other emissions, of course) are usually visualized by means of frequency-time spectrograms displaying the time evolution of the wave power spectral density. Among the various lightning-induced phenomena, whistlers [Eckersley, 1935; Storey, 1953; Helliwell, 1965], ion cyclotron whistlers [Smith et al., 1964; Gurnett et al., 1965], subprotonospheric whistlers [Carpenter et al., 1964; Kimura, 1966; Chum et al., 2009], magnetospherically reflected whistlers and in particular the so-called Nu-whistlers [Smith and

\footnotetext{
${ }^{1}$ Space Research Institute, Russian Academy of Sciences, Moscow, Russia.

${ }^{2}$ Problems of Physics and Energetics Department, Moscow Institute of Physics and Technology, Moscow, Russia.

${ }^{3}$ Cucuron, France.

${ }^{4}$ Institute of Atmospheric Physics, Academy of Sciences of the Czech Republic, Prague, Czech Republic.

${ }^{5}$ Faculty of Mathematics and Physics, Charles University, Prague, Czech Republic.

${ }^{6}$ LPC2E/CNRS, Orléans, France.

${ }^{7}$ Apatity Division, Polar Geophysical Institute, Apatity, Russia.

Corresponding author: D. R. Shklyar, Space Research Institute, Russian Academy of Sciences, Profsoyuznaya Str. 84/32, 117997 Moscow, Russia. (david@iki.rssi.ru)

C2012. American Geophysical Union. All Rights Reserved. 0148-0227/12/2012JA018016
}

Angerami, 1968; Shklyar et al., 2004] are among the most familiar. The appearance of spectrograms showing lightninginduced phenomena depends strongly on the frequency-time resolution [Storey, 1953], as well as on the total time interval covered. Thus, for instance, a detailed spectrogram of whistlers usually includes a few seconds of observations. As was shown by Eckersley [1935], the frequency-time curve of a whistler trace in a spectrogram is determined by the frequency dependence (or dispersion) of the group velocity of whistler mode waves. For detailed VLF spectrograms, the orbital motion of the satellite (if the spectrogram is of data taken onboard a satellite) is of minor importance.

[3] Besides detailed spectrograms, overview spectrograms are often used; these are calculated for time intervals of the order of minutes or even tens of minutes. In such a case, satellite motion is of major importance, and the spectrogram is determined both by wave propagation and by satellite motion; in other words, the spectrogram represents the evolution of the wave spectrum in space as well as time. Spectrograms containing oblique noise bands [Chum et al., 2003] and wedge-like spectrograms [Shklyar et al., 2010] exemplify wave events that originate in lightning strokes as whistlers do, but which need overview spectrograms to display their essential features.

[4] In this paper we study ELF events - proton whistlers and short-fractional-hop whistlers - in the frequency band $f \lesssim 1000 \mathrm{~Hz}$, observed by the DEMETER satellite in equatorial latitudes at altitudes around $660 \mathrm{~km}$. These events originate in lightning strokes, and we focus on observations 
made in the hemisphere opposite to that of the source lightning; whistlers observed in this situation are termed "transequatorial" [Watanabe and Ondoh, 1976; Bošková et al., 1976]. In contrast to proton whistlers observed in the same hemisphere as the source lightning, trans-equatorial proton whistlers last much longer, for about $10 \mathrm{~s}$, and the upper cutoff frequency of these signals is close to the equatorial, not the local, proton gyrofrequency. (In the present context, the term "equatorial" means the value of a quantity at the magnetic equator on the field line passing through the point where these events were observed, not its value at the point where their waves crossed the equator).

[5] Proton whistlers were discovered by Smith et al. [1964] in the data from the Injun 3 and Alouette satellites. Their formation was explained by Gurnett et al. [1965], who made a comprehensive study of ion cyclotron wave propagation in the multicomponent ionospheric plasma. This study concerned the proton whistlers described by Smith et al. [1964], which were observed as upgoing waves in the same hemisphere as the lightning source. The effects of collisions and of the latitudinal variation of the geomagnetic field on ion cyclotron whistlers were discussed by Jones [1969]. A theoretical approach to the problem of ion cyclotron whistlers based on a full-wave solution of Maxwell's equations has recently been developed by Ferencz et al. [2001].

[6] Proton whistlers propagating to the point of observation from the opposite hemisphere across the geomagnetic equator (i.e. trans-equatorial or downgoing proton whistlers) were first found in VLF data from the Interkosmos 5 satellite by Likhter et al. [1974], who also established that in this case the upper cutoff frequency is equal to the proton equatorial gyrofrequency. Watanabe and Ondoh [1976, 1980] described upgoing and downgoing deuteron whistlers found in the VLF data from ISIS 1 and ISIS 2 at low geomagnetic latitudes. More observations of these phenomena on Interkosmos 5, 14, and 19 , together with their detailed investigation based on calculations of characteristic frequencies in plasma containing four types of ions $\left(\mathrm{O}^{+}, \mathrm{He}^{+}, \mathrm{H}^{+}, \mathrm{D}^{+}\right)$were presented by Bošková et al. [1984] and Bošková and Jiříček [1986].

[7] In this paper we address the problem of proton whistlers on the basis of new 6-component ELF wave measurements from the DEMETER satellite. The kind of multicomponent analysis that we employ has been first developed by Storey and Lefeuvre [1974, 1979, 1980]. An application can be seen in Santolik and Parrot [1999]. Some representative spectrograms are presented and discussed in section 2 . The results of ray tracing calculations that reveal the features of ion cyclotron wave propagation in a multicomponent plasma are given in section 3. They enable us to explain the main properties of the measured spectrograms, and this explanation, supported by numerical simulations of the spectrograms, is presented in section 4 .

\section{DEMETER Observations of Band-Limited Spectra Below the Proton Gyrofrequency}

[8] DEMETER was a French satellite with a nearly circular orbit; it was launched in June 2004, and operated until the end of 2010. Originally the altitude of the orbit was about $710 \mathrm{~km}$, but it was reduced to about $660 \mathrm{~km}$ in December, 2005. The data of interest for the present study were acquired while operating in the so-called "burst mode", when the waveforms of all six electromagnetic field components were measured at the sampling frequency of 2500 Hz [Berthelier et al., 2006; Parrot, 2006; Parrot et al., 2006]. From these simultaneous measurements of the six components we constructed a $6 \times 6$ electromagnetic spectral matrix, which we used as input to some of the existing methods of wave analysis [Santolik et al., 2003, 2006].

[9] An example of the DEMETER data in the proton cyclotron frequency band is shown in Figure 1, along with the results of the wave analysis. Orbital data for the satellite pass include universal time (UT), geomagnetic latitude $\left(\lambda_{m}\right)$, McIlwain parameter $(L)$, geographic latitude (Lat), and geographic longitude (Lon). This figure presents a set of overview spectrograms. The first and second panels are the frequencytime spectrograms for the total (i.e., the sum over three components) power spectral density of the electric and magnetic field fluctuations, respectively. A set of discrete brief electromagnetic wave events can be seen, each with a well defined gap in its spectrum, roughly $100 \mathrm{~Hz}$ wide and beginning around $400 \mathrm{~Hz}$; the reason why this gap exists is given in section 4 . The frequency of the bottom of the gap decreases as time goes on, following the local proton cyclotron frequency at the spacecraft, which is plotted as the dashed black line. These events were caused by a sequence of lightning strokes, each of which excited a fractional-hop whistler and a proton whistler. The lower parts of the spectrograms, below the gap, are full of proton whistlers. They have a sharp upper frequency cutoff at the local proton gyrofrequency. The average duration of a proton whistler trace is $\lesssim 2$ seconds, which can be estimated from detailed spectrograms such as those shown in Figure 2. Above the gap, the upper parts of these spectrograms are full of fractional-hop whistlers.

[10] Results for the wave polarization and for the directions of the wave normal and of the Poynting vector, from analysis of simultaneous measurements of the six field components, are shown in the third through fifth panels, each of which represents the frequency-time dependence of one of these wave properties. (The format which is being described is used in all figures of this type, namely, Figures 1-4.) The third panel shows the ellipticity of the magnetic field fluctuations: the absolute value corresponds to the ratio of the minor to the major polarization axes, the sign corresponds to the sense of polarization, negative to left-handed and positive to right-handed [Santolik et al., 2002]. Note the change of wave polarization from right- to left-handed at crossover frequency at which the waves are linearly polarized (see Figure 5 below for details). The fourth panel gives the polar angle of the wave normal vector with respect to the ambient magnetic field determined from the wave magnetic field data [Santolik et al., 2003]. The values range from $0^{\circ}$ to $90^{\circ}$ because, from these data alone, it is not possible to distinguish between a wave propagating in a given direction and one propagating in the opposite direction. This ambiguity is resolved in the fifth panel which shows the component of the Poynting vector parallel to the Earth's magnetic field, normalized with respect to its standard deviation; positive values correspond to directions at acute angles to the ambient magnetic field, and negative values to directions at obtuse angles to the field [Santolik et al., 2001]. The analysis was restricted 

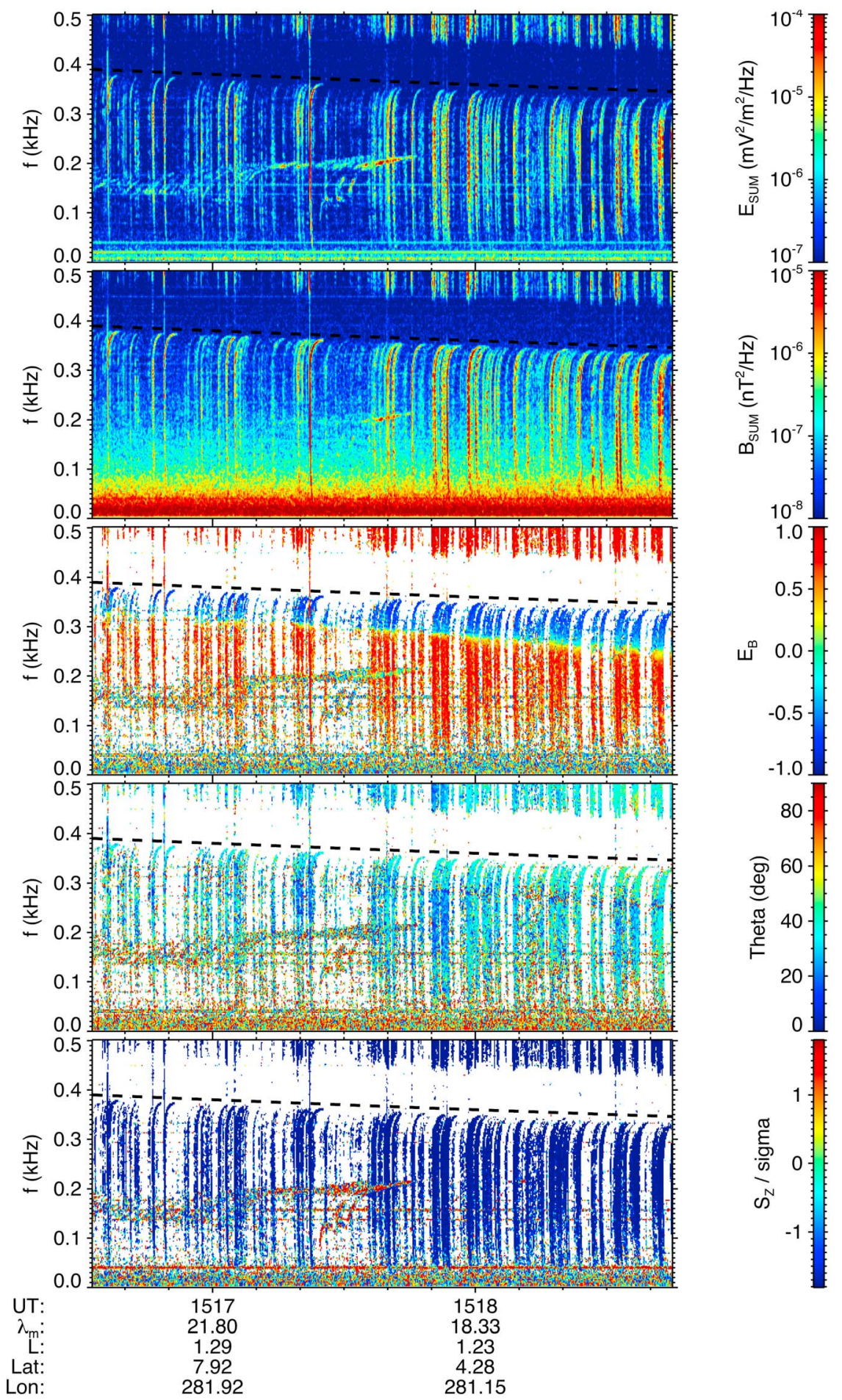

Figure 1. DEMETER observations of the proton cyclotron frequency band, containing proton whistlers and the lower-frequency portions of fractional-hop electron whistlers. The data were acquired on September 12,2008 . The meaning of the individual panels is (from the top down): power spectral density of electric field fluctuations; power spectral density of magnetic field fluctuations; sense of polarization (the red and blue colors correspond to right- and left-handed polarization, respectively); polar angle of the wave vector with respect to the ambient magnetic field; and normalized parallel component of the Poynting vector. 

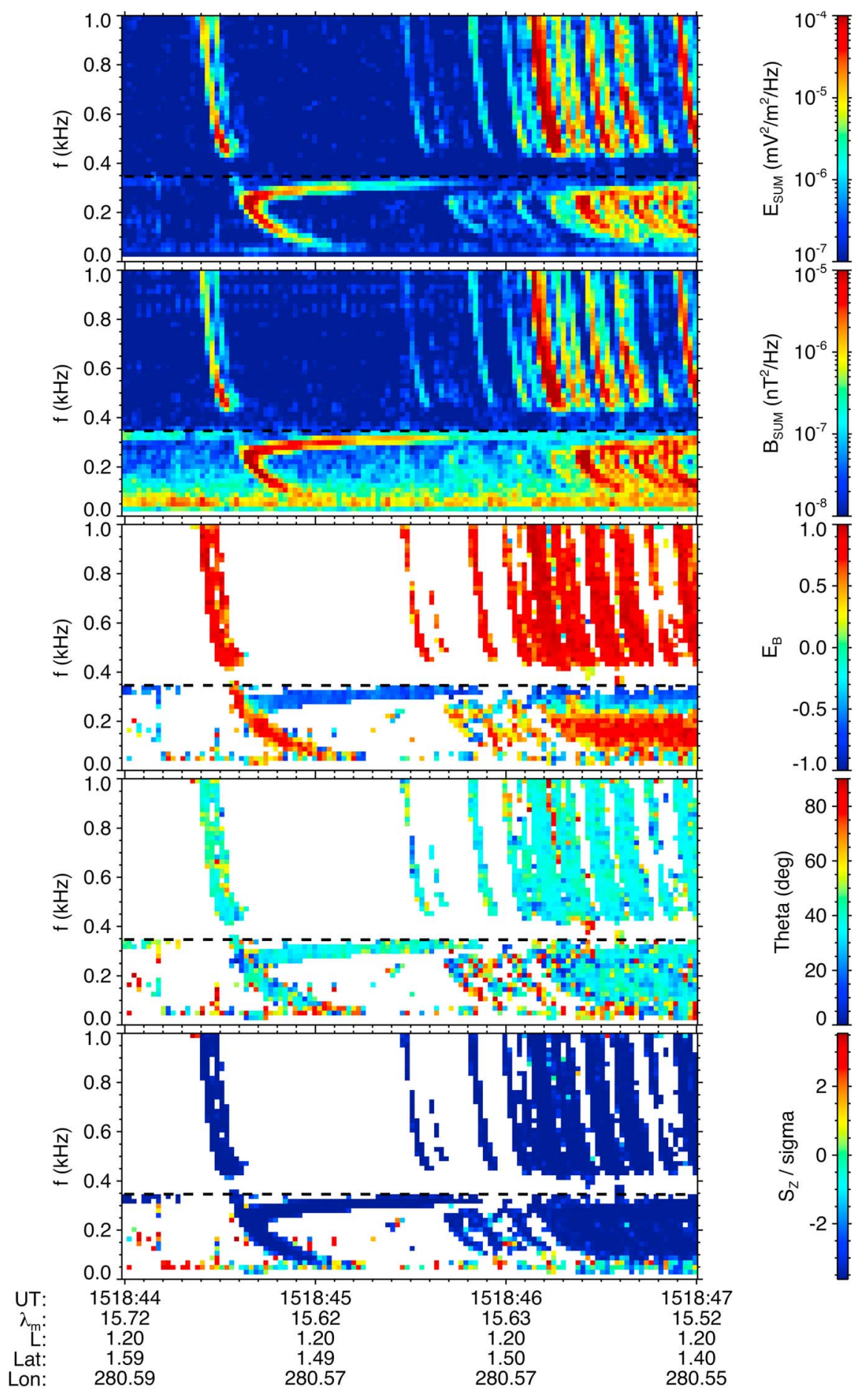

Figure 2. Detailed spectrogram of one of the proton whistlers shown in Figure 1. Three seconds of measurements are zoomed to provide a better time resolution.

to those frequency-time intervals where the power spectral density of the electric field fluctuations was larger than $2 \times$ $10^{-7} \mathrm{mV}^{2} \mathrm{~m}^{-2} \mathrm{~Hz}^{-1}$ and the power spectral density of the magnetic field fluctuations was larger than $2 \times 10^{-8} \mathrm{nT}^{2}$ $\mathrm{Hz}^{-1}$, in order to select good specimens of the events of interest. It can be seen that parallel component of the Poynting vector is negative. Since the parallel components of the wave and the Poynting vectors have the same sign, we conclude that the same is true for the wave vector. The data for Figures 1 and 2 were taken in the northern hemisphere, where the Earth's magnetic field is directed downward, so these facts show that the observed events originated below the satellite, in the same hemisphere. We should mention that waves propagating in the opposite direction, thus coming 


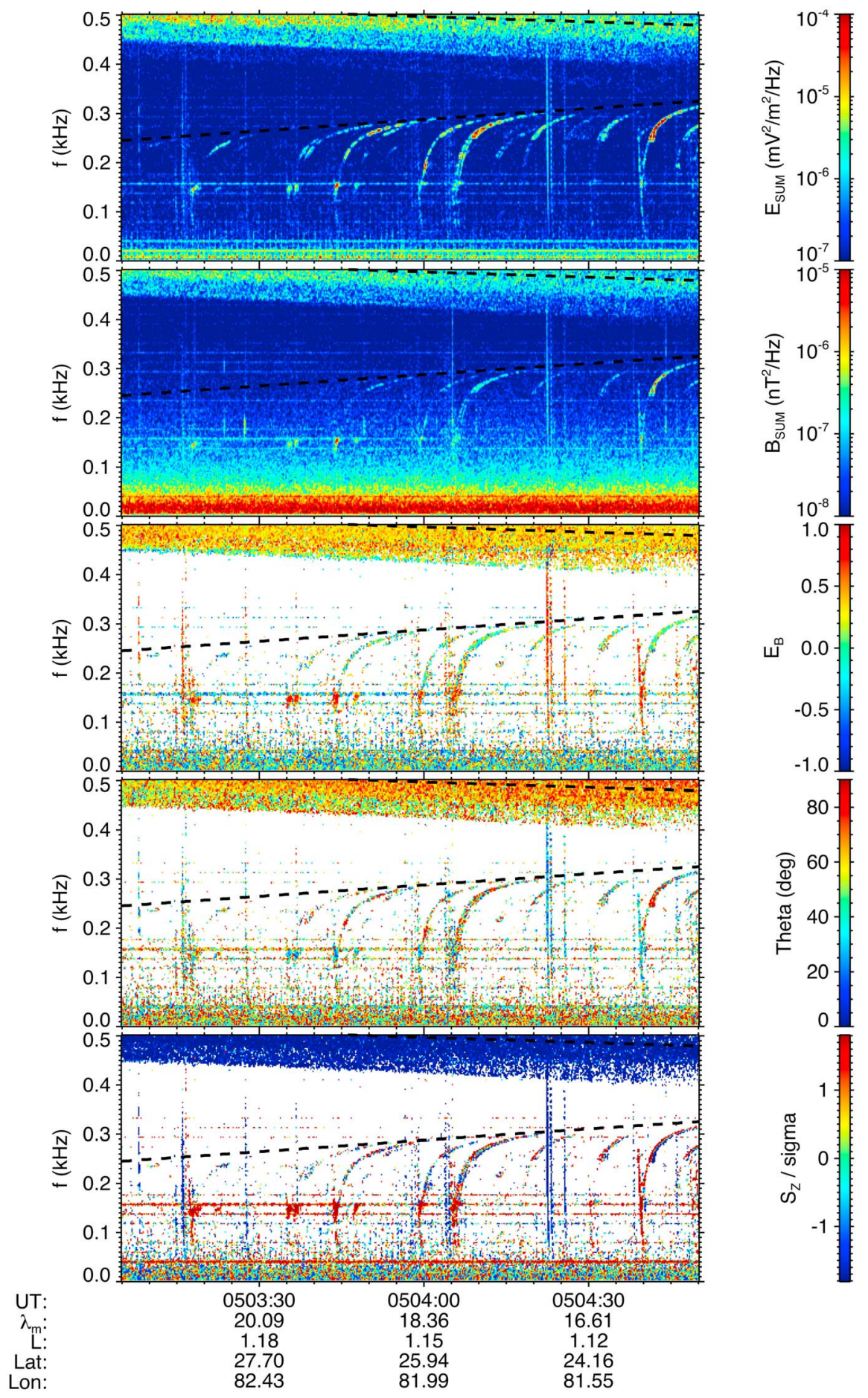

Figure 3. DEMETER observations of the proton cyclotron frequency band in the northern hemisphere. These data were taken on November 30, 2005. The format is the same as in Figure 1. The lower dashed line - which is not the same as the one shown in Figure 1-is a plot of the equatorial proton cyclotron frequency at a given $L$-shell.

from the opposite hemisphere, and having spectra with upper cutoff frequencies well below the local proton cyclotron frequency, can also be seen in the left part of Figure 1.

[11] Waves propagating in the whistler mode at frequencies well above the proton gyrofrequency are observed in the hemisphere opposite to the causative lightning stroke as 1_whistlers (in the ionosphere) or as one-hop whistlers (on the ground). As was mentioned in section 1, the first observations of proton whistlers in the hemisphere opposite to the lightning stroke were reported by Likhter et al. [1974] and Bošková et al. [1976]. Examples of such observations made by the DEMETER satellite are given in Figures 3 and 4 . 


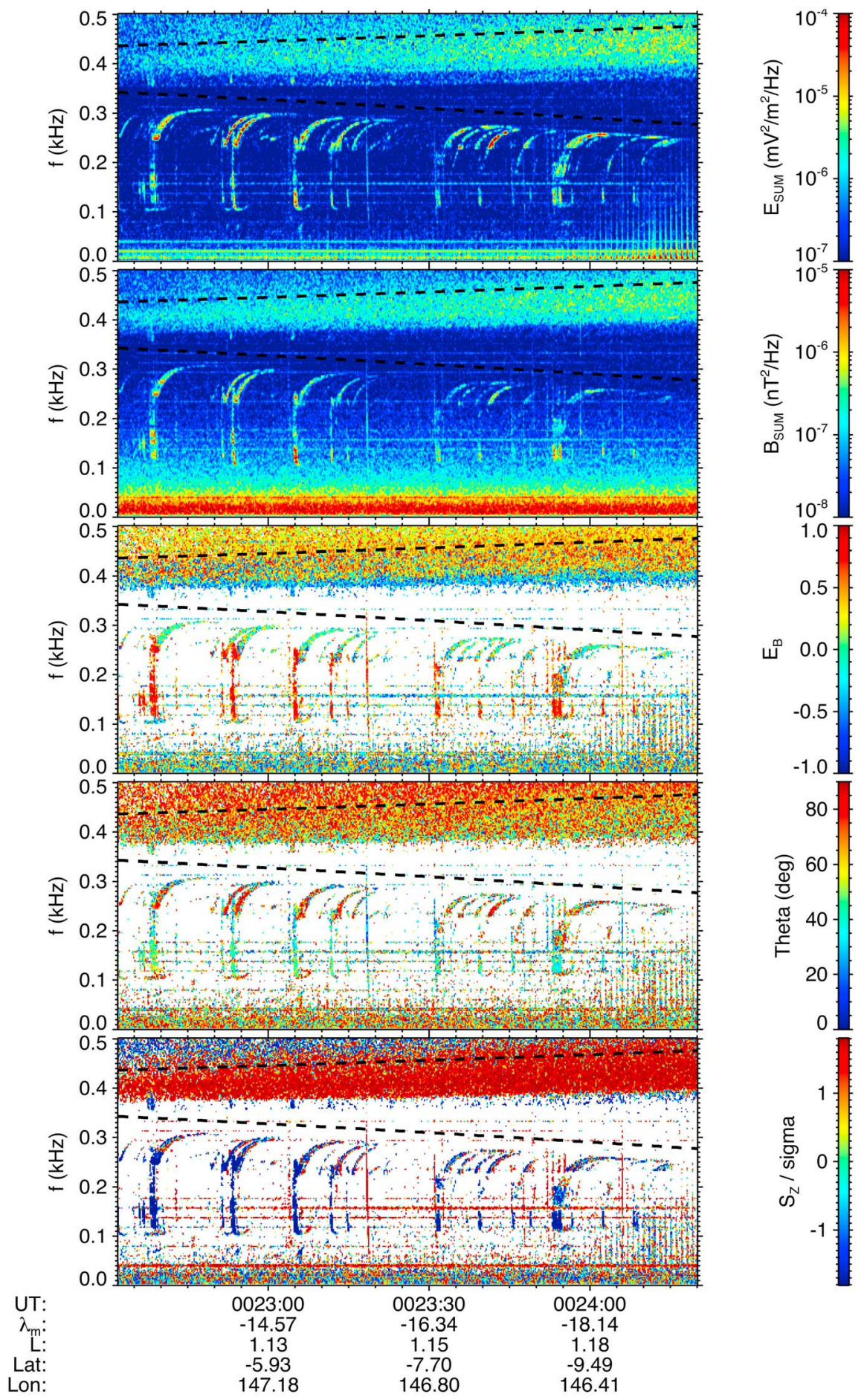

Figure 4. DEMETER observations of the proton cyclotron frequency band in the southern hemisphere. These data were taken on May 13, 2005. The format is the same as in Figure 1.

The format is the same as in Figure 1. Moreover, in each of the figures, the lower dashed line is a plot of the equatorial proton gyrofrequency versus $L$, while the upper dashed line shows, as before, the variation of the local proton gyrofrequency along the satellite path. In contrast to the proton whistlers observed in the same hemisphere as the source lightning, the typical time duration of a single trace is distinctly larger, of the order of $10 \mathrm{~s}$ (determined from a detailed frequency-time plot, not shown). The increase in the trace duration is caused by the frequency dependence of the group velocity, which is most pronounced close to the equatorial proton gyrofrequency. In agreement with the earlier studies [see, e.g., Likhter et al., 1974; Bošková et al., 1976; Bošková and Jiřčcek, 1986], the upper cutoff 
frequency is related to the equatorial proton cyclotron frequency at the given $L$-shell. Note that since the orbit of the DEMETER spacecraft is circular, the equatorial cyclotron frequency on the $L$-shell of the spacecraft decreases as a function of the absolute value of the geomagnetic latitude. Consequently the change of the upper cutoff frequency observed by DEMETER is related to the geomagnetic latitude of the spacecraft. In the case of the event shown in Figure 3 the spacecraft is in the northern hemisphere and is moving toward the geomagnetic equator - i.e. to lower Lvalues - so the upper cutoff frequency increases. In much the same way, in the event shown in Figure 4 the spacecraft is in the southern hemisphere and is moving away from the equator, to larger negative latitudes, and the upper cutoff frequency decreases accordingly. The propagation of electromagnetic wave events observed in this lower band of the frequency spectrum, and the explanation of fine features of the latter, are the main subjects of the present paper. We prove that the events depicted in these spectrograms are due to proton cyclotron waves originating in the opposite hemisphere, while the waves propagating in the second mode, which is the continuation of the electron whistler mode below the proton gyrofrequency, have essentially different trajectories which do not reach the satellite. In this respect, the spectrograms under discussion differ from those of wave events observed in the same hemisphere as the lightning strokes, where waves in both modes are observed simultaneously as proton whistlers and as fractional-hop whistlers (see Figure 1 and Smith et al. [1964] and Gurnett et al. [1965]).

[12] The conjecture that the phenomena observed in Figures 3 and 4 are coming from the opposite hemisphere is experimentally supported by the results of the wave analysis. Specifically, parallel component of the Poynting vector and, thus, of the wave vector in Figure 3 are positive, corresponding to a component of propagation along the ambient magnetic field. Given that it was recorded in the northern hemisphere, this means that the wave is coming from lower latitudes, as would correspond to the propagation from the opposite, southern hemisphere. Similarly, parallel component of the Poynting vector in Figure 4 is negative, corresponding to a component of propagation opposite to the ambient magnetic field. Since this event was recorded in the southern hemisphere, it again corresponds to propagation from the oppositenow the northern - hemisphere.

[13] Apart from the obvious differences between the spectrograms shown in Figure 3 and Figure 4, we should mention some less conspicuous ones, namely: the traces shown in Figure 3 terminate closer to the equatorial proton gyrofrequency than the traces shown in Figure 4, which, in addition, have a more pronounced curvature at the upper cutoff. The maximal frequencies on the traces measured at the same latitudes differ by about $20-30 \mathrm{~Hz}$. In section 4, we will return to these peculiarities of the spectrograms.

[14] Examination of the traces that form transequatorial ion cyclotron whistlers shown in Figures 3 and 4 shows that, generally, in the upper frequency parts the wave polarization is almost linear, while the wave normal angles are close to $\pi / 2$. In the lower frequency parts, the waves are right-hand polarized and have large enough wave normal angles, although not too close to $\pi / 2$ (see the third and fourth panels of the above mentioned figures). This picture of wave propagation, along with the observed wave properties, will be theoretically analyzed and simulated in the next sections. Note that the observations also show finer effects which are out of scope of this paper. For example, results of analysis at short timescales (not shown) reveal that most of the transequatorial proton whistler main traces are followed by fainter reflected components. This complex propagation pattern also causes occasional deviation from the above described regular results in the cases where the assumption of the presence of a single plane wave becomes invalid.

\section{Propagation Features of Ion Cyclotron Waves in the Upper Ionosphere}

[15] As was pointed out in the pioneering paper on ion cyclotron whistlers [Gurnett et al., 1965] and confirmed by later work on this subject, in order to understand spectrograms observed on a satellite in the frequency band below the proton gyrofrequency, the multicomponent nature of the ionospheric plasma must be taken into account. This point is illustrated by Figure 5 which represents the roots of the cold plasma dispersion relation, i.e., the values of the refractive index squared, $N^{2}=k^{2} c^{2} / \omega^{2}$, as functions of the wave frequency for four wave normal angles $\theta=5^{\circ}, 30^{\circ}, 60^{\circ}$, and $85^{\circ}$, when the plasma contains three types of ions. Only positive values of $N^{2}$ corresponding to propagating waves are shown. The displayed quantity $\ln \left(1+N^{2}\right)$ is a monotonic function of $N^{2}$, but it depends differently on $N^{2}$ in different ranges of $N^{2}$ in ways that enable us to display the whole frequency band on the same plot.

[16] In discussing the wave propagation and polarization, we will adopt a Cartesian coordinate system in which the ambient magnetic field $\mathbf{B}_{0}$ is directed along the $Z$-axis, while the wave normal vector lies in the $(X, Z)$-plane. From Figure 5 we see that when the wave frequency $\omega \rightarrow\left(\omega_{\mathrm{H} \alpha}-0\right)$, i.e., when $\omega$ approaches, from below, the cyclotron frequency $\omega_{\mathrm{H} \alpha}$ of each type of ion, remaining less than $\omega_{\mathrm{H} \alpha}$, one root $N^{2}(\omega, \theta)$ of the dispersion relation tends to $+\infty$, while the second root stays finite and positive. Thus, at these frequencies, there are two propagating modes. As is known from the general theory of wave propagation in a cold magnetoplasma, waves for which $N^{2} \rightarrow \infty$ are quasielectrostatic $(B \ll N E$ in CGS units,) and their electric field is linearly polarized along the wave normal [Ginzburg and Rukhadze, 1972]. When $\omega \rightarrow\left(\omega_{\mathrm{H} \alpha}+0\right)$, one root tends to $-\infty$, which corresponds to the non-propagating mode, while the second root, which remains finite and positive, corresponds to a propagating wave. The wave polarization is indicated in Figure 5 by colors: the blue and red colors correspond to left- and righthand polarized waves, respectively, with ellipticity shown by darkness, namely, the lighter is the color, the smaller is the ratio of the minor to the major polarization axes, so that the darkest colors correspond to circular polarization. We see that on the same branch of the solution, which we associate with a particular wave mode, the sense of polarization changes at the so-called crossover frequencies, where the two branches of solutions come closest to each other. One can show that at this frequency the off-diagonal components of the dielectric tensor vanish, as does the $E_{y}$ component of the wave electric field, so again the wave is linearly polarized. In this case, however, in contrast to the case where $N^{2} \rightarrow \infty$, the $E_{z}$ component of the wave electric field remains small; thus the wave is polarized almost along the $x$-axis regardless of the direction 
Three types of ions: $\mathrm{H}^{+}(25 \%), \mathrm{He}^{+}(5 \%), \mathrm{O}^{+}(70 \%)$
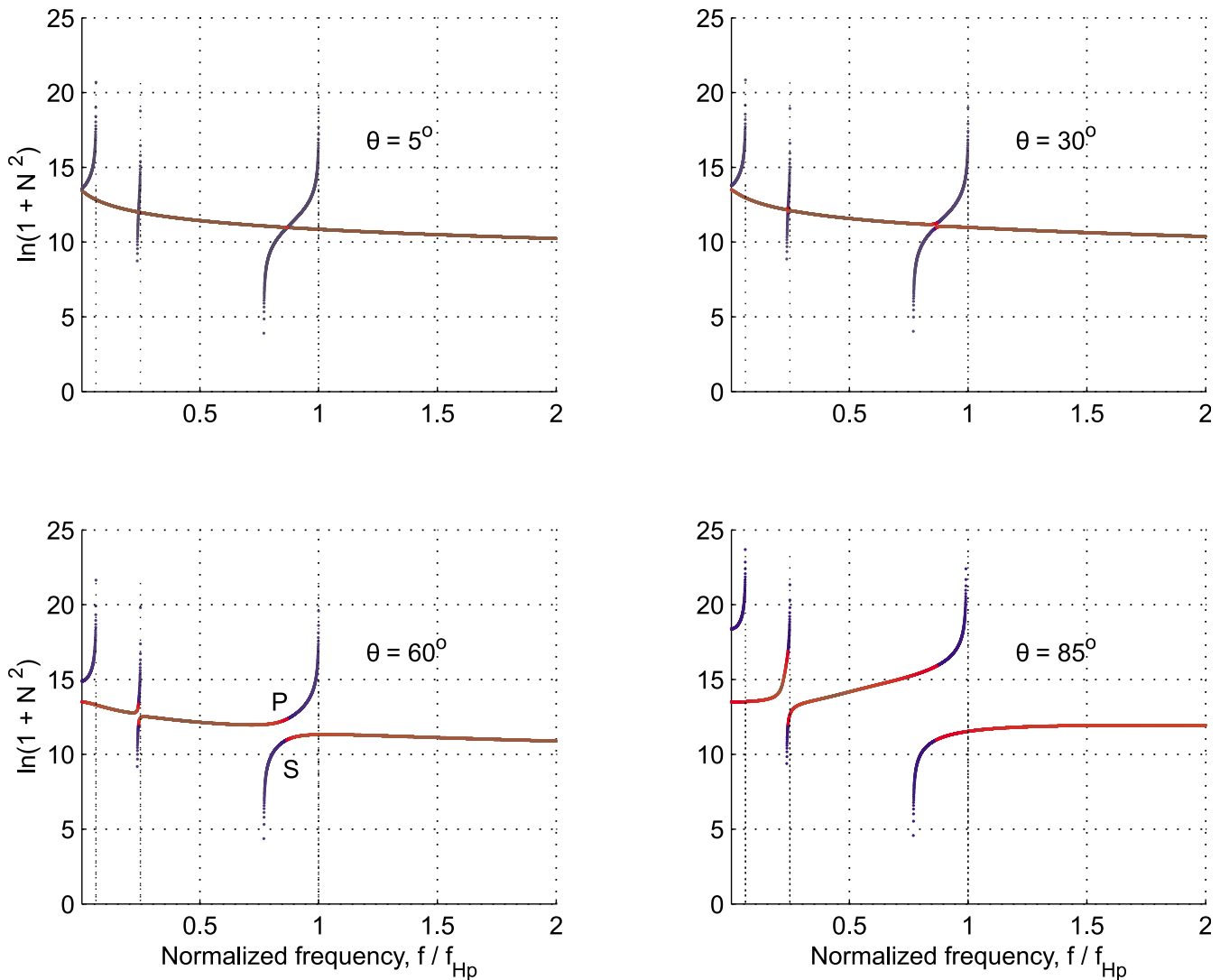

Figure 5. Presentation of $N^{2}$ and wave polarization as a function of normalized wave frequency for 4 fixed wave normal angles. Left- or right-handed polarization corresponds to blue or red color, respectively, with the darkness indicating the ellipticity: the darker are the colors, the closer the polarization is to circular.

of the wave normal. Since the components of the dielectric tensor depend on the plasma parameters, but are independent of the wave normal angle, the same is true for the crossover frequency normalized with respect to the cyclotron frequency. We should stress that the fact that both waves are linearly polarized at the crossover frequency [Gurnett et al., 1965] by no means implies that the refractive index has the same value for both wave modes. As for the wave magnetic field, the corresponding polarization ellipse lies in the plane perpendicular to $\mathbf{k}$-vector, while the ellipticity is indicated in Figure 5 by color intensity as described above.

[17] In the following discussion we will distinguish between three concepts: wave phenomena (proton whistlers, fractionalhop whistlers, etc.), wave polarization, and wave mode (defined above as a continuous branch of the solution to the dispersion equation), which are sometimes mixed up. Over certain parts of the dispersion curves a wave may be characterized by its sense of polarization, or may be classified as "fast" (having lower refractive index) or "slow" (having higher refractive index) wave, but, as is obvious from Figure 5, none of these properties can define a wave mode since each property changes along the dispersion curve corresponding to a given mode; we should mention that this is not the case in a plasma containing only one type of positive ion, such as an electronproton plasma.
[18] We will be mainly interested in the two wave modes in the band of frequencies centered on the proton cyclotron frequency, where obliquely propagating waves can excite the proton cyclotron resonance. The branches of the curves representing these two modes are labeled "P" and "S" on Figure 5 (bottom left). In the frequency band below proton gyrofrequency, where two propagating modes exist simultaneously, "P" waves correspond to larger values of the wave refractive index. When the subject matter concerns both of these branches, we will use the term "ion cyclotron waves" in this collective sense. The P-mode waves have a resonance $\left(N^{2} \rightarrow \infty\right)$ at $\omega=\omega_{\mathrm{H} p}$ and a low-frequency cutoff $\left(N^{2}=0\right)$ below the helium gyrofrequency $\omega_{\mathrm{H}_{\mathrm{He}}}$. The S-mode waves have a low-frequency cutoff between the helium and proton gyrofrequencies; its exact position depends on the ion composition. In particular, when oxygen is the dominant ion, this cutoff frequency is close to the proton gyrofrequency.

[19] In contrast to the case of a multicomponent plasma, in an electron-proton plasma both branches continue down to $\omega=0$ without cutoffs or changes of polarization. At $\omega \ll$ $\omega_{\mathrm{H} p}$, the left- and right-hand polarized waves are called Alfvén and magnetosonic waves, respectively. The branch that we call S-mode continues to higher frequencies and becomes the whistler mode, which has a resonance close to 


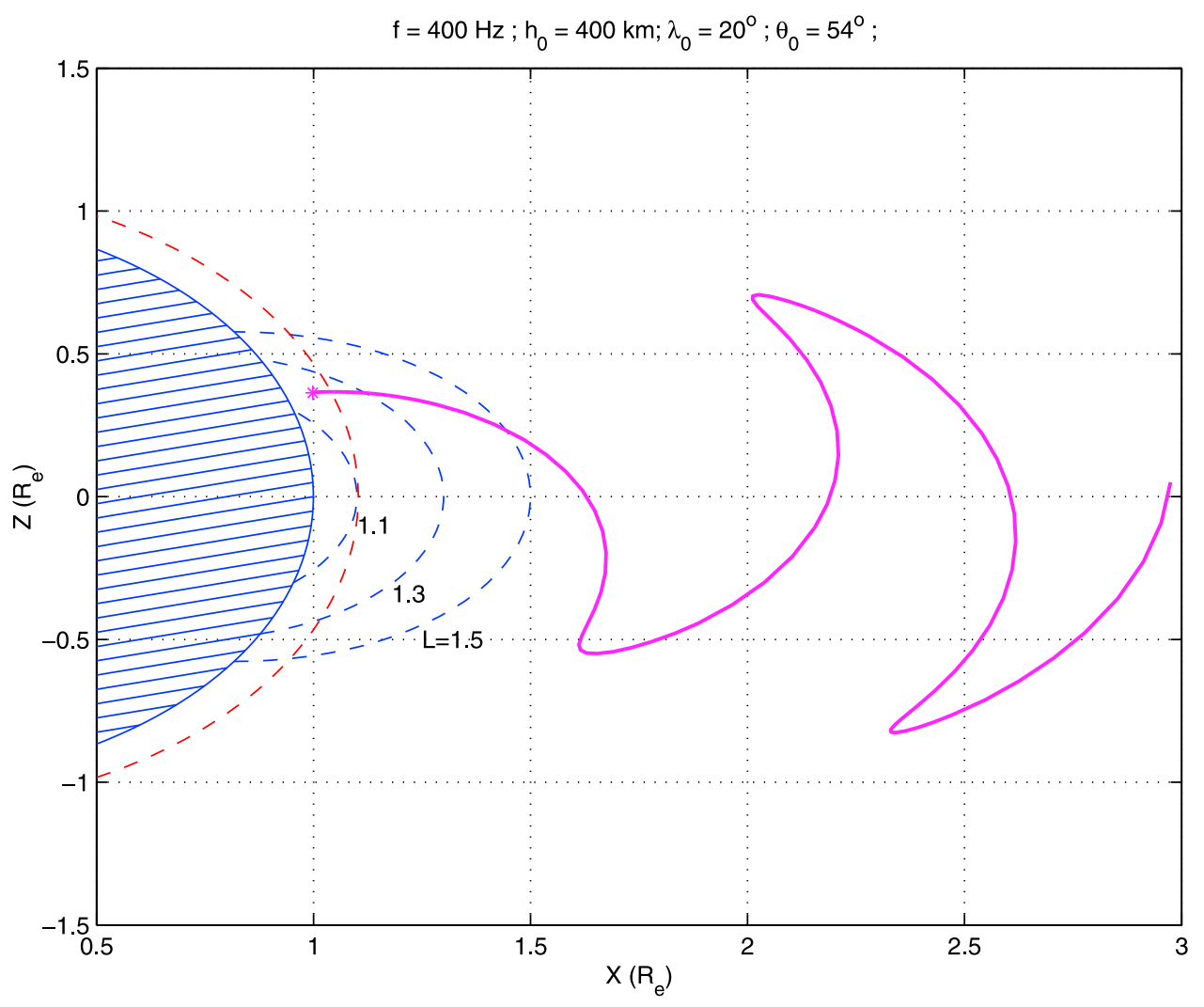

Figure 6. Ray trajectory for a $400 \mathrm{~Hz}$ S-mode wave.

$\min \left(\omega_{\mathrm{H} e}, \omega_{\mathrm{P} e}\right) \cos \theta$ where $\omega_{\mathrm{He}}$ and $\omega_{\mathrm{P} e}$ are the electron gyro and plasma frequencies, respectively.

[20] The foregoing discussion is in line with the work of Gurnett et al. [1965], which we have found to be consistent with our observations, notably with the lower frequency cutoffs and the changes of polarization. We should repeat, however, that a different theoretical explanation for the formation of ion whistlers has been advanced by Ferencz et al. [2001]. Further research, observational as well as theoretical, is needed to resolve any disagreements that may exist between the predictions of these two theories.

[21] The consideration in this paper concerns the region above the level of maximum electron density in the ionosphere, where the propagation of ion cyclotron waves (ICW) under discussion can be described by geometrical optics. Using the same arguments as for whistler mode waves, based on Snell's law, we may assume that the wave normal angle at the altitude of $\gtrsim 350-400 \mathrm{~km}$, where we start the ray tracing, is close to the vertical. At low initial latitudes $\lambda_{0} \sim$ $15-20^{\circ}$, the initial angle between the vertical wave normal and the ambient magnetic field is $\sim 50-60^{\circ}$. Details of the ray tracing calculations are given in Appendix A.

[22] The trajectory of an ion cyclotron wave in the meridian plane is determined by the initial coordinates (latitude and altitude), wave frequency, initial direction of the wave normal vector, and the branch of the solution to the dispersion equation. In the frame of geometrical optics (GO), a solution of the GO equations always remains on the same branch; in other words, the wave mode does not change, though the wave polarization does. Mode conversion that connects the wave modes in the case of a strong mode coupling is a purely wave effect. This effect does not play a significant role for large wave normal angles $\theta$ because the values of the refractive index for the two wave modes at the crossover frequency are markedly different (see Figure 5).

[23] A typical trajectory of an S-mode wave is shown in Figure 6; the starting point is marked by an asterisk. In this and the following figures of this type, the coordinates $X$ and $Z$ are normalized to the Earth's radius $R_{e}$. The trajectory is quite similar to those of whistler mode waves below the lower hybrid resonance frequency, which is not surprising, of course, since the S-mode and the whistler mode represent the same branch of the solution to the dispersion equation. The red dashed line in Figure 6 represents the DEMETER orbit. We see that, on low-orbiting satellites like DEMETER, $\mathrm{S}$ waves can be observed only when the satellite is in the same hemisphere as the lightning stroke, and the wave frequency is above the lower-frequency cutoff at the starting point.

[24] For $\mathrm{P}$ waves with frequencies below the proton gyrofrequency, but well above the lower cutoff at their starting point, there are two different types of trajectories: those that run upward to the opposite hemisphere, then turn down and descend, and then oscillate between two hemispheres; and those that stick in the source hemisphere, never reaching the opposite one. Examples of ray trajectories corresponding to $200 \mathrm{~Hz}$ waves starting at different latitudes are shown in Figure 7. In this and the following figures of this type, the blue dashed lines correspond to $L$-shells 1.1 , 1.3 , and 1.5, and the green dashed line gives the isoline of the proton gyrofrequency $\omega_{\mathrm{H} p}(L, \lambda)=\omega$ where $\omega$ is the wave frequency under consideration. We see that, in order to reach the opposite hemisphere, the wave must have a sufficiently 

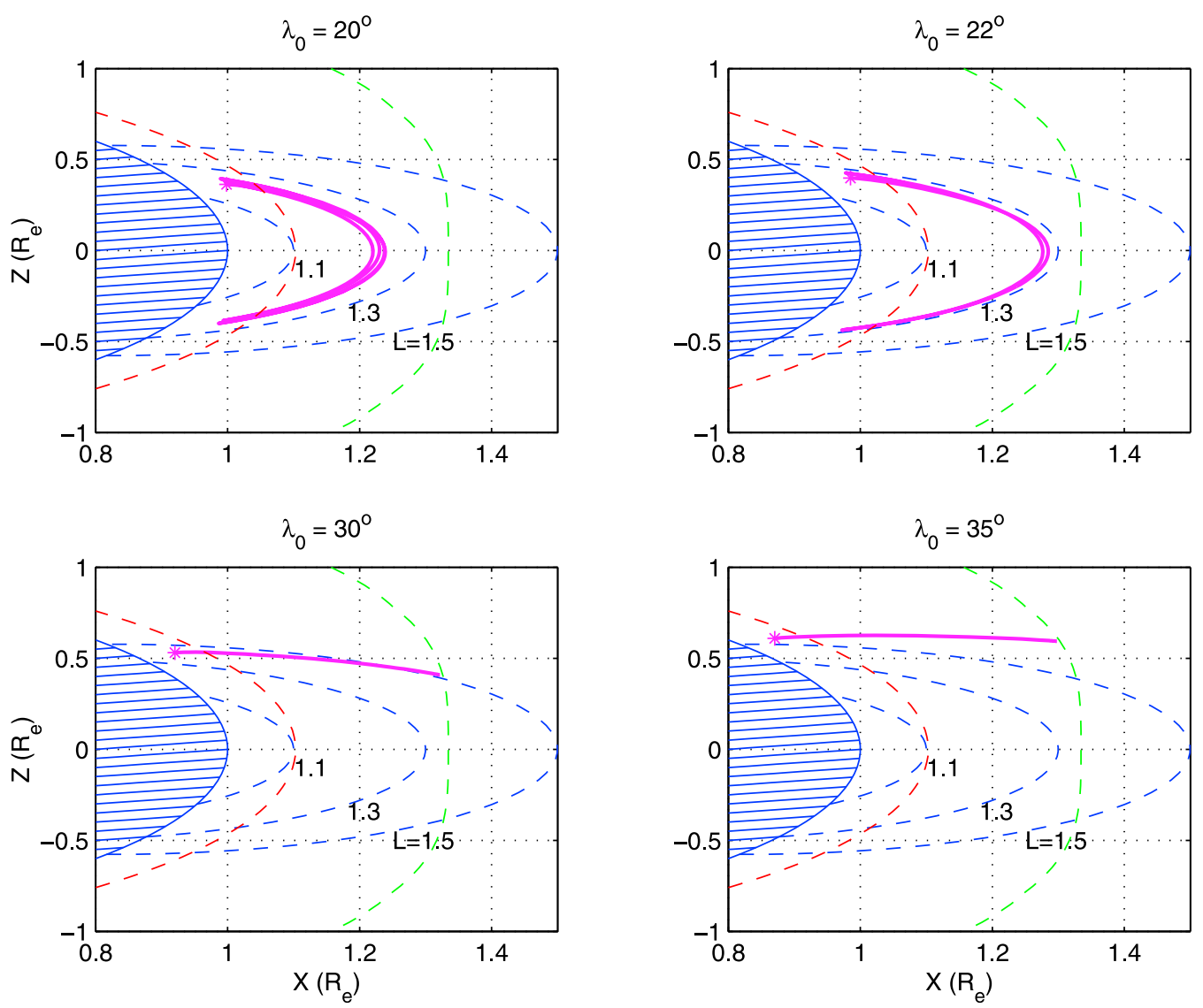

Figure 7. Ray trajectories for proton cyclotron waves of $200 \mathrm{~Hz}$ and various initial latitudes.

low initial latitude. For the wave frequency of $200 \mathrm{~Hz}$ and the chosen plasma parameters, the limiting initial latitude is $\sim 23.5^{\circ}$. This limiting latitude decreases with increasing frequency. Thus, for instance, for $250 \mathrm{~Hz}$ waves, the maximum initial latitude from which the wave reaches the opposite hemisphere is $\sim 19.5^{\circ}$. As can be seen from Figure 7 and as is confirmed by similar calculations for different wave frequencies and initial latitudes, for waves that reach the opposite hemisphere the final latitudes at DEMETER altitudes $(\sim 660 \mathrm{~km})$ are close to being geomagnetically conjugate to the initial ones. Besides depending on the initial latitude, the ray trajectories depend strongly on the wave frequency. This dependence is illustrated by Figure 8. Detailed examination of the ray trajectories shows that, for waves starting at the same latitude and reaching the opposite hemisphere, the bending of the trajectory is more pronounced for the higher-frequency waves, with the result that they reach the satellite closer to the equator. At the same time, as the simulations show, the frequency band of the waves that reach the satellite in the opposite hemisphere contracts with increasing initial latitude. We emphasize once again that the wave mode, which is determined by the branch of dispersion relation, always remains the same along the ray trajectory, though the wave polarization may change.

[25] The variations of the wave parameters along ray trajectories of the two types mentioned above are shown in Figures 9 and 10. The graphs in the left column show (from top to bottom): variations of $L$-shell, wave normal angle $\theta$, and parallel component of the group velocity (Figure 9) or its magnitude (Figure 10), while the graphs in the right column display the variations of magnetic latitude $\lambda$, refraction index, and polarization of the wave magnetic field: positive and negative values corresponding to rightand left-handed polarization, respectively, while the magnitude gives, as before, the ratio of the minor to major polarization axes. For the wave that reaches the opposite hemisphere and then oscillates between two hemispheres, the variations of all quantities, but the $L$-shell, has a quasiperiodic character. The wave normal angle oscillates around $90^{\circ}$, the index of refraction increases on the average, and polarization is left-handed on the most parts of the ray trajectory, while around the reflection points the polarization becomes right-handed. The reflection of the P-mode wave takes place in the regions where the wave frequency is equal to hybrid resonance frequency determined by the relation $\varepsilon_{1}=0$ (see (A5)), which is illustrated by the Figure 9 (top right) where the quantity proportional to $\varepsilon_{1}$ is plotted by the magenta dashed line. This reflection is similar in nature to the well known lower hybrid resonance (LHR) reflection first discovered theoretically by Kimura [1966]. The LHR frequency is also determined by the relation $\varepsilon_{1}=0$ which has solutions in various frequency domains [Stix, 1992]. Similar to LHR reflection, the reflection of the P-mode wave corresponds to $k_{\|}=v_{g \|}=0$.

[26] For the wave which sticks in the same hemisphere all parameters change monotonically (see Figure 10). In this case the wave normal angle is far from $90^{\circ}$ which is consistent with the known fact that (at least in an electron-proton 

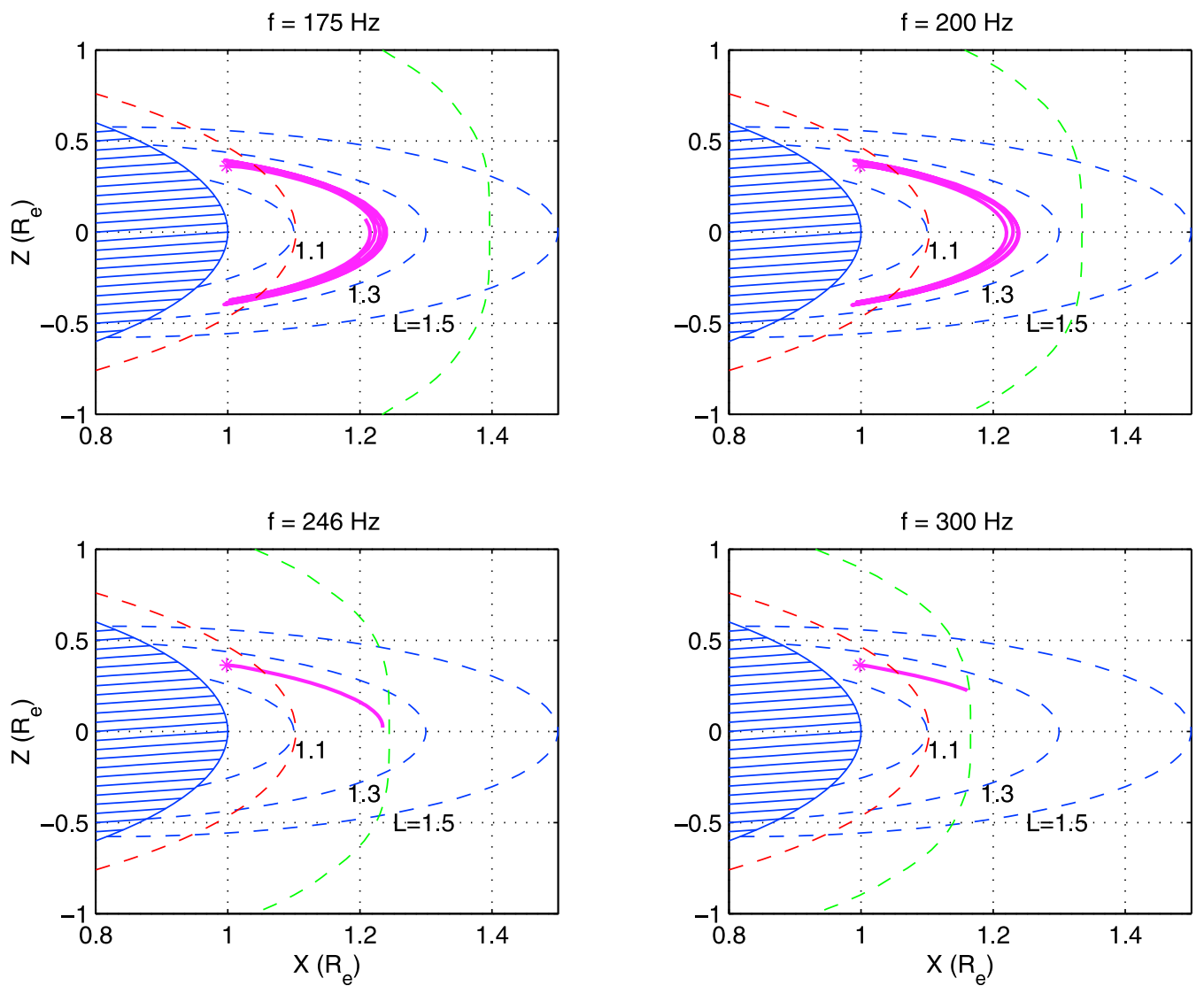

Figure 8. Ray trajectories for proton cyclotron waves of various frequencies, starting from $\lambda_{0}=20^{\circ}$.

plasma) in the frequency range of proton cyclotron waves, when $\omega \simeq \omega_{\mathrm{H} p}$, the resonance occurs for all angles $\theta$ not close to $90^{\circ}$, while when $\omega<\omega_{\mathrm{H} p}$, which should be the case for waves that cross the equator and reach the opposite hemisphere, the resonance occurs for $\theta \simeq 90^{\circ}$ [see, e.g., Ginzburg and Rukhadze, 1972]. It is worth mentioning that for waves that get stuck, the wave refractive index increases and the magnitude of the group velocity decreases so fast that the distance covered during infinite time remains finite. These waves should be absorbed in the background plasma. When the wave frequency decreases, while remaining above the helium gyrofrequency, the character of trajectories remains the same as shown in Figures 7 (top) and 8 (top), but the altitude of the reflection point increases in such a way that the waves do not reach low altitudes, and thus they cannot be received on low-orbiting satellites.

\section{Explanation and Numerical Modeling of ICW Spectrograms}

[27] The propagation features of ion cyclotron waves described above and illustrated by Figures 6-10 enable us to explain the ICW spectrograms under discussion as follows. When a spectrogram is taken of an event observed in the same hemisphere as the lightning stroke, both S-and P-mode waves are observed. Since P waves have a resonance at the proton gyrofrequency, the local value of the latter is the upper cutoff frequency for this band, at which the time delay of the trace is greatest. On the contrary, S waves have no resonance in the considered frequency band, but they do have a cutoff at a lower frequency. As was mentioned above, in a multicomponent plasma where $O^{+}$ions are dominant, this cutoff frequency is close to the proton gyrofrequency. Thus, the frequency of S-mode waves should always be above the proton gyrofrequency $\omega_{\mathrm{H} p}$ in the lower ionosphere, the maximum value of which determines the lower cutoff frequency. Since $\omega_{\mathrm{H} p}$ decreases with altitude, and since $\mathrm{P}$ waves have an upper frequency cutoff at the local gyrofrequency, there is a gap between the resonance cutoff of $\mathrm{P}$ waves and the lower frequency cutoff of $\mathrm{S}$ waves. Note that the latitudinal dependence of both cutoff frequencies follows the latitudinal dependence of $\omega_{\mathrm{H} p}$, as can be seen in Figure 1.

[28] When a spectrogram is taken of an event observed in the opposite hemisphere, the situation changes significantly. As was mentioned above, S-mode waves do not reach the satellite in this case, and the spectrum is formed only by Pmode waves. In order to reach the satellite, a wave has to cross the equator, thus its frequency should be below the equatorial proton gyrofrequency on its ray trajectory. To reach the satellite, the waves of different frequencies originating from one lightning stroke start from slightly different initial latitudes and propagate along relatively close trajectories. In general, the higher frequency waves, if they reach the opposite hemisphere, experience stronger bending toward the equator than the lower frequency waves, thus higher frequency waves that reach the satellite start from 

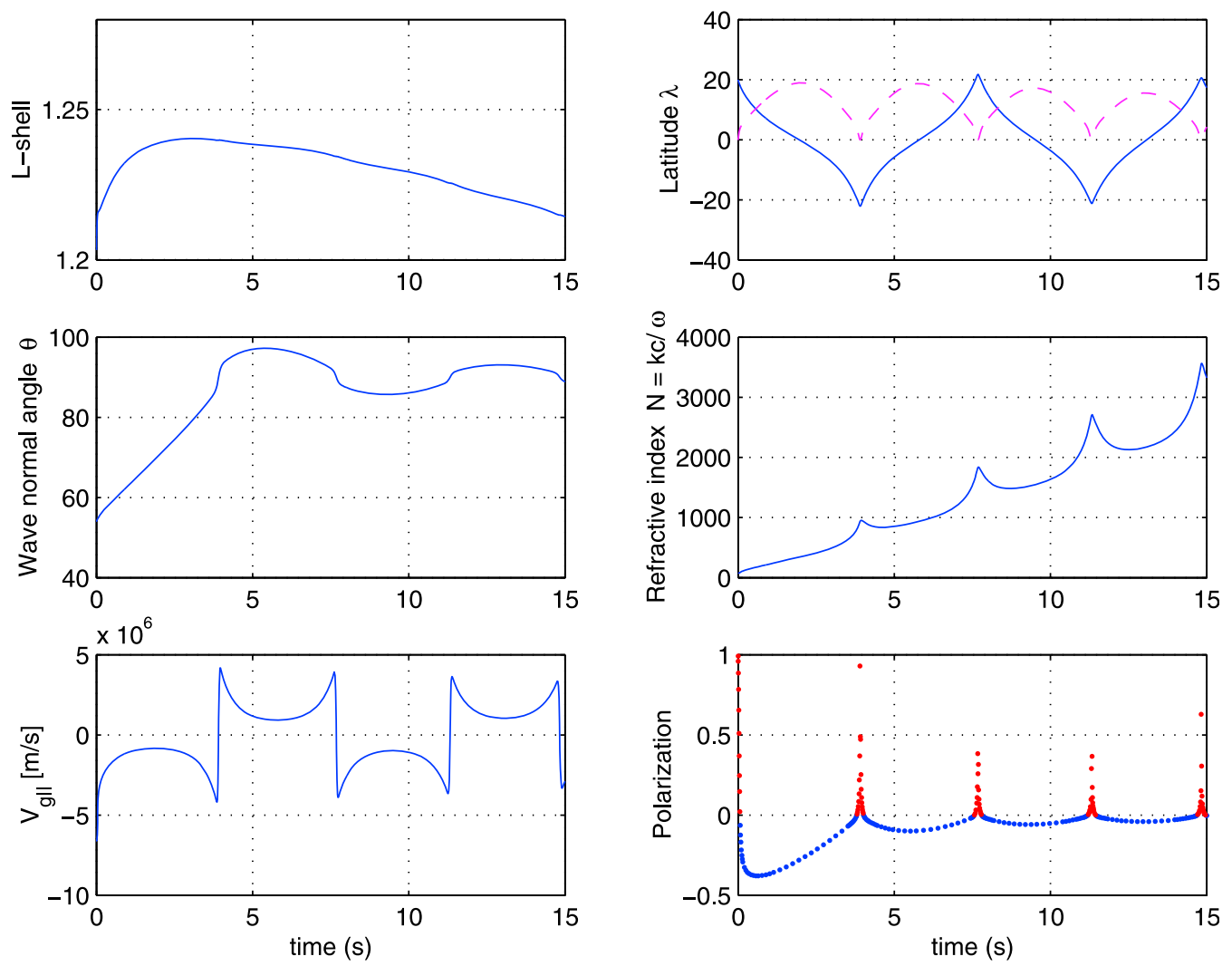

Figure 9. Wave parameters: $L$-shell, wave normal angle $\theta$, parallel group velocity, latitude $\lambda$, refractive index $N=k c / \omega$, and magnetic field polarization for a $200 \mathrm{~Hz}$ wave that starts at $\lambda_{0}=20^{\circ}$ and reaches the satellite altitude in the opposite hemisphere.

higher latitudes as compared to lower frequency waves. However, for every latitude of the satellite, there is an upper limit to the frequency that can reach the satellite, because the higher frequencies either come down at lower latitudes or get stuck before crossing the equator. This limiting frequency is subject to a rule: the higher the wave frequency, the lower is the maximal accessible latitude in the opposite hemisphere, or, equivalently, the higher this latitude, the lower is the maximal frequency. This reasoning explains the latitudinal dependence of the upper frequency cutoff in Figures 3 and 4.

[29] As was shown in section 3, the features of the ray trajectories described above refer to a finite frequency range below the equatorial proton gyrofrequency. With a further decrease of the wave frequency, the property of the ray trajectories changes in a sense that the down-coming waves are reflected before they reach the satellite altitudes. This fact explains the lower-frequency cutoff in the spectrograms in Figures 3 and 4.

[30] The results obtained above also explain most of the peculiarities of the spectrograms shown in Figures 3 and 4 and mentioned in section 2. As the ray tracing shows, the waves that form the upper frequency part of these spectrograms have their wave normal angles close to $90^{\circ}$ and very large values of refractive index $N \sim 3000$. At the latitude $\lambda \sim 20^{\circ}$, the angle between the vertical and the ambient magnetic field is $\sim 54^{\circ}$. Given the circular trajectory of the DEMETER satellite, the angle between the wave normal vector $\mathbf{k}$ and the satellite velocity $\mathbf{v}_{S}$ appears to be also close to $54^{\circ}$. Then, the Doppler shift $\Delta f=-\mathbf{k} \cdot \mathbf{v}_{s} \sim \pm 12 \mathrm{~Hz}$, its sign depending on the direction of the satellite: it is positive when the satellite moves toward the equator and negative when the satellite moves toward a pole. This explains why the traces shown in Figure 3 (where the satellite is moving toward the equator) terminate closer to the proton equatorial gyrofrequency than the traces shown in Figure 4 (where the satellite is moving toward the pole). The difference in maximum frequencies measured on the satellite is the double Doppler shift and is of the order of $20-30 \mathrm{~Hz}$. Since the refractive index becomes very large close to the highest frequency in the trace, the Doppler shift is predominantly responsible for the trace bending; some part must be due to the variation of the equatorial proton gyrofrequency along the orbit, but rough calculations have shown that its contribution to the trace bending, though of the correct sign, is much smaller than that due to the Doppler effect.

[31] One reservation must be expressed in this connection. While the ray tracings indicate that the wave normal angles $\theta$ of the trans-equatorial whistlers should be close to $90^{\circ}$ near their upper frequency cutoffs, and the spectrograms in Figures 3 and 4 show Doppler shifts that bear out this prediction, the values determined for these angles by analysis of the wave magnetic field data (fourth panels in Figures 3 and 4) are not close to $90^{\circ}$. This discrepancy related presumably to the presence of reflected waves (see the end of Section 2) has not yet been explained.

[32] The ray tracing calculations described in section 3 and in Appendix A make possible numerical simulation of 

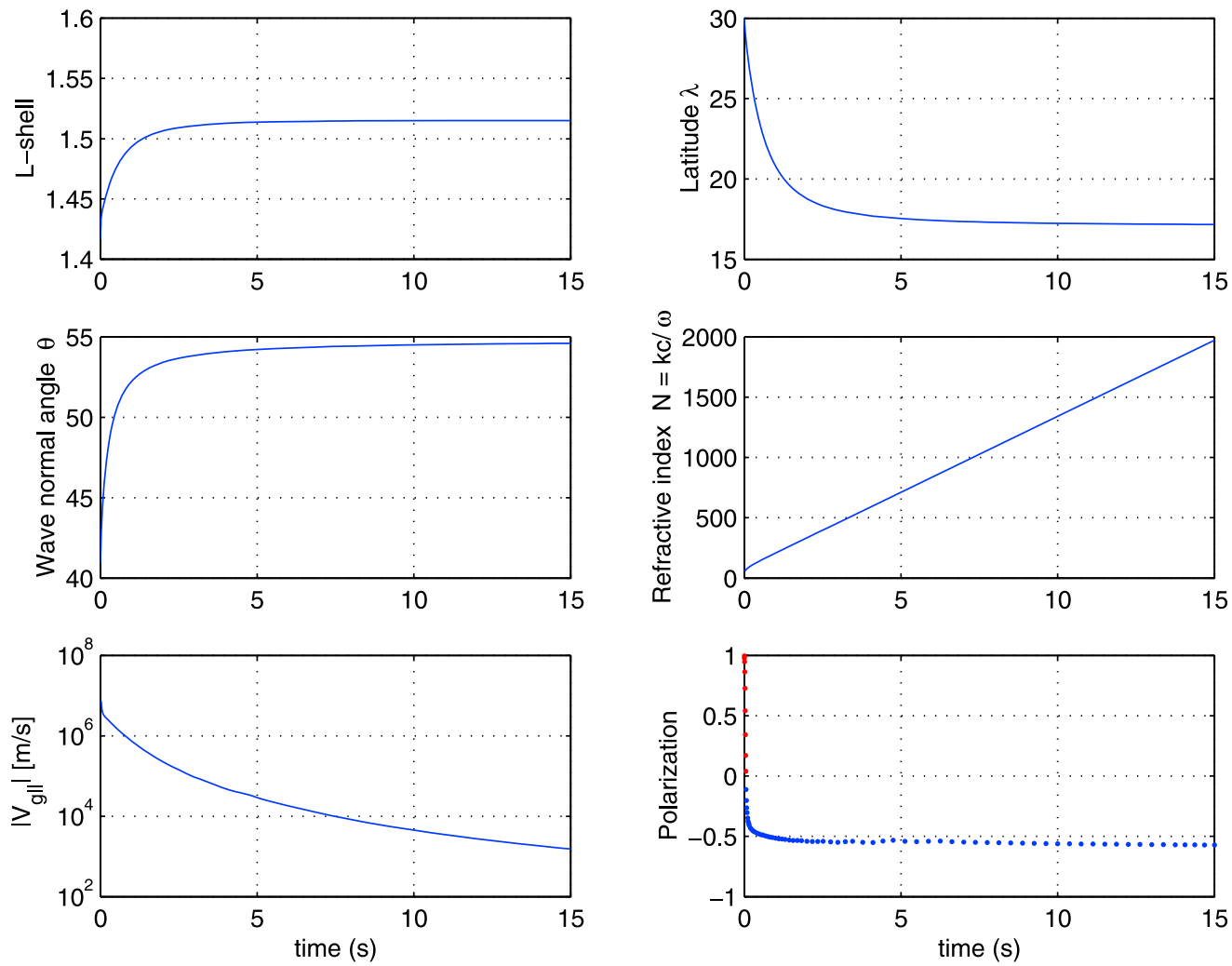

Figure 10. Wave parameters: $L$-shell, wave normal angle $\theta$, magnitude of parallel group velocity, latitude $\lambda$, refractive index $N=k c / \omega$, and magnetic field polarization for $200 \mathrm{~Hz}$ wave that starts at $\lambda_{0}=$ $30^{\circ}$ and gets stuck in the source hemisphere.

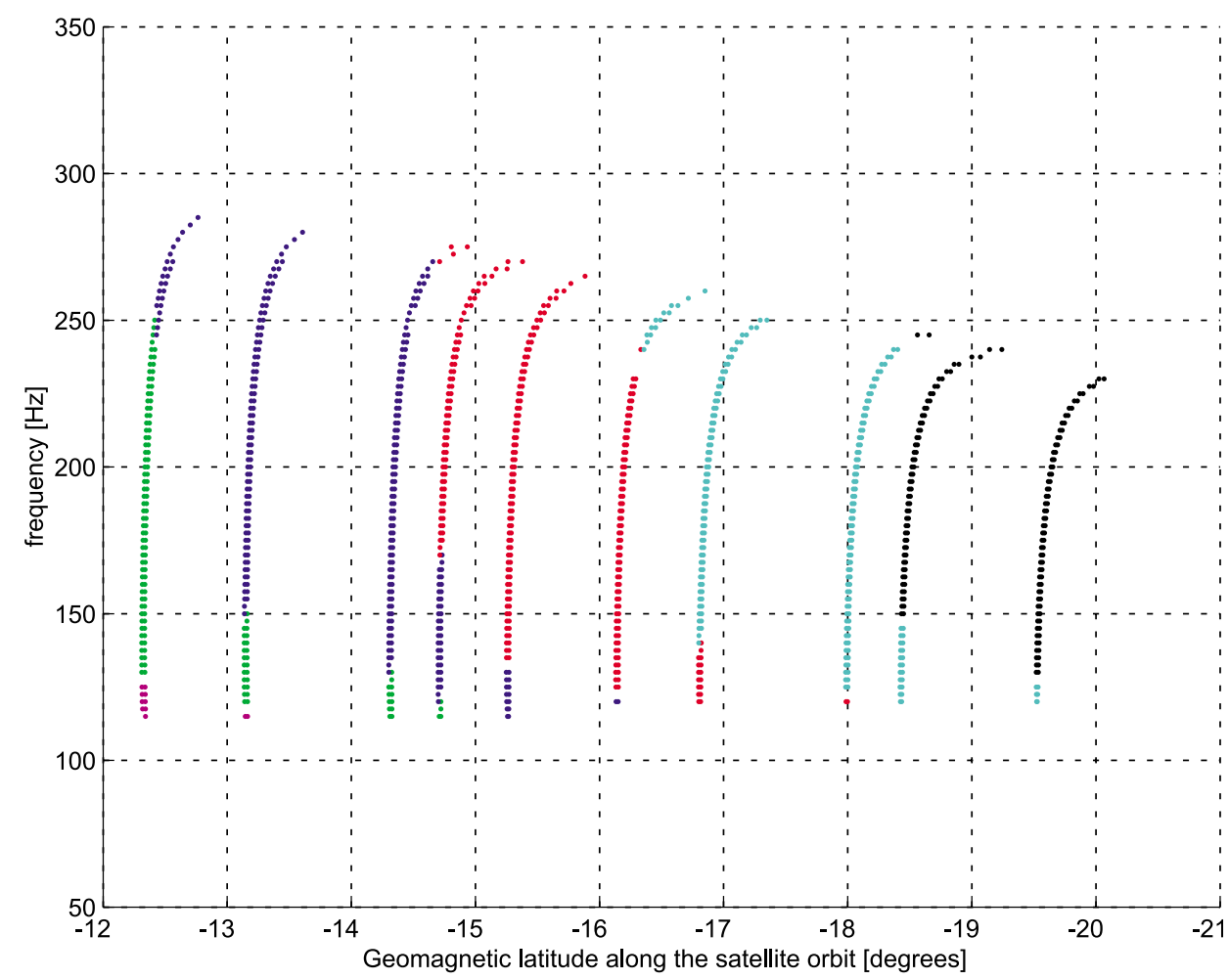

Figure 11. Simulated overview spectrogram of transequatorial proton whistlers which models the real spectrogram shown in Figure 4. Satellite moves toward the pole in the S hemisphere. Thunderstorm is in the $\mathrm{N}$ hemisphere. 
the spectrograms of wave events observed onboard the DEMETER satellite. The method of spectrogram modeling is described in detail in Shklyar and Jiřiček [2000], Shklyar et al. [2004], and Shklyar [2005]. Figure 11 shows an example of simulated overview spectrogram of transequatorial proton whistlers for the case when the satellite moves in the hemisphere opposite to the lightning sources. In fact, the figure presents only the frequency-time plots; the spectral intensity is not shown. Instead, the initial latitudes from which the different frequencies come to the satellite are indicated by colors. The intervals of initial latitude $\left(12.5-13.8^{\circ}\right),(13.8-$ $\left.15.2^{\circ}\right),\left(15.2-16.5^{\circ}\right),\left(16.5-17.8^{\circ}\right),\left(17.8-19.2^{\circ}\right),\left(19.2-20.5^{\circ}\right)$ correspond to the colors magenta, green, blue, red, cyan, and black, respectively. As can be seen, the simulated spectrogram reproduces qualitatively the main features of the real one: upper- and lower-frequency cutoffs, characteristic frequency band, and the latitudinal span of observation. However, they do not show the peculiarities due to the Doppler effect, mentioned earlier in this section; this effect was not included in the simulations. Comparison of the spectral intensity in real and simulated spectrograms will be the subject of further work.

\section{Concluding Remarks}

[33] Ion cyclotron whistlers, upgoing as well as downgoing (trans-equatorial), have been studied, both experimentally and theoretically, since their discovery by Smith et al. [1964], Likhter et al. [1974]. Basic ideas explaining these wave phenomena have been put forward by Gurnett et al. [1965] and developed in further studies by various authors (see section 1). In this paper we have investigated proton whistlers observed by DEMETER using 6-component measurements of the wave electromagnetic field. This measurement technique, in conjunction with the appropriate analysis, yields important wave characteristics such as the wave normal vector (thus, the index of refraction), the Poynting flux, and the wave polarization, which were unavailable with earlier measurements. We have presented the results of this analysis that permit to determine experimentally the direction of wave arrival and the mode of propagation, as well as to explain a fine point of the observed spectra, namely the dependence of the upper cutoff frequency on the direction of satellite motion, which we attribute to Doppler shift. The experimental results have been supplemented by the ray tracing analysis of ion cyclotron wave propagation in multicomponent plasma in the upper ionosphere, and the corresponding numerical modeling of spectrograms. By these means, we have explained and reproduced several important features of proton whistlers, such as their presence only on low $L$-shells $(L \lesssim 1.2)$, the observable frequency band and its dependence on satellite position.

\section{Appendix A: Equations and Details of the Ray Tracing}

[34] As is well known, ray tracing consists of solving the equations of geometrical optics as applied to a certain dispersion equation. The latter expresses the wave frequency as a function of the wave normal vector, and of the variables entering the dispersion relation through the spatial dependence of the ambient magnetic field and plasma parameters. If the dispersion relation for a given wave mode is known in an explicit form, i.e. $\omega=H(\mathbf{k}, \mathbf{r})$, then the equations of geometrical optics have the form:

$$
\frac{d \mathbf{r}}{d t}=\frac{\partial H}{\partial \mathbf{k}} ; \quad \frac{d \mathbf{k}}{d t}=-\frac{\partial H}{\partial \mathbf{r}} ;
$$

Equations (A1) have Hamiltonian form with canonically conjugate variables $(\mathbf{r}, \mathbf{k})$. Further on, we consider 2D geometry with the Cartesian coordinates shown in Figures 6 8 , and we assume that all waves propagate in the magnetic meridian plane which coincides with the $(X, Z)$-plane of our coordinate system.

[35] An explicit form of the dispersion relation for ion cyclotron waves in multicomponent plasma is not known, and we have to deal with its implicit form set by the general dispersion relation for electromagnetic waves in a cold magnetoplasma, which can be written as follows [see, e.g., Ginzburg and Rukhadze, 1972]:

$$
F \equiv A k^{4}+B \frac{\omega^{2}}{c^{2}} k^{2}+C \frac{\omega^{4}}{c^{4}}=0,
$$

with

$$
\begin{aligned}
& A=\varepsilon_{1} \sin ^{2} \theta+\varepsilon_{3} \cos ^{2} \theta ; \quad C=\varepsilon_{3}\left(\varepsilon_{1}^{2}-\varepsilon_{2}^{2}\right) ; \\
& B=-\varepsilon_{1} \varepsilon_{3}\left(1+\cos ^{2} \theta\right)-\left(\varepsilon_{1}^{2}-\varepsilon_{2}^{2}\right) \sin ^{2} \theta,
\end{aligned}
$$

$\varepsilon_{1,2,3}$ being the non-zero components of the dielectric tensor $\varepsilon_{i j}$ :

$$
\varepsilon_{i j}(\omega)=\left(\begin{array}{ccc}
\varepsilon_{1} & i \varepsilon_{2} & 0 \\
-i \varepsilon_{2} & \varepsilon_{1} & 0 \\
0 & 0 & \varepsilon_{3}
\end{array}\right)
$$

given by

$$
\begin{aligned}
& \varepsilon_{1}=1-\sum_{s} \frac{\omega_{\mathrm{P} s}^{2}}{\omega^{2}-\omega_{\mathrm{H} s}^{2}} ; \quad \varepsilon_{2}=-\sum_{s} \frac{\omega_{\mathrm{H} s} \omega_{\mathrm{P} s}^{2}}{\omega\left(\omega^{2}-\omega_{\mathrm{H} s}^{2}\right)} ; \\
& \varepsilon_{3}=1-\sum_{s} \frac{\omega_{\mathrm{P} s}^{2}}{\omega^{2}}
\end{aligned}
$$

where the summation is over all plasma species: electrons and all types of ions. After inserting (A3) into (A2), the function $F$ that defines the dispersion relation (A2) appears to be a function of $k_{\perp}^{2}=k^{2} \sin ^{2} \theta, k_{\|}^{2}=k^{2} \cos ^{2} \theta$, and of the coordinates $(X, Z)$, which are not canonically conjugate with $\left(k_{\perp}, k_{\|}\right)$. That is why, in order to write the equations of geometrical optics in Hamiltonian form, we need to change to new canonically conjugate variables, for which we choose dipolar coordinates $(L, M)$ and the corresponding momenta $\kappa_{\mathrm{L}}, \kappa_{\mathrm{M}}$. These quantities are expressed through $(X, Z)$ and $\left(k_{\perp}, k_{\|}\right)$as follows:

$$
L(X, Z)=\frac{1}{R_{\mathrm{E}}} \frac{\left(X^{2}+Z^{2}\right)^{3 / 2}}{X^{2}} ; \quad M(X, Z)=R_{\mathrm{E}}^{2} \frac{Z}{\left(X^{2}+Z^{2}\right)^{3 / 2}},
$$

$$
\kappa_{\mathrm{L}}=h_{\mathrm{L}} k_{\perp} ; \quad \kappa_{\mathrm{M}}=h_{\mathrm{M}} k_{\|},
$$

where $R_{\mathrm{E}}$ is the Earth's radius, while $h_{\mathrm{L}}, h_{\mathrm{M}}$ are Lamé coefficients related to the dipolar coordinates $L$ and $M$, respectively:

$$
h_{\mathrm{L}}=\frac{R_{\mathrm{E}}}{(1+\Lambda)(1+4 \Lambda)^{1 / 2}} ; \quad h_{\mathrm{M}}=\frac{L^{3} R_{\mathrm{E}}}{(1+\Lambda)^{5 / 2}(1+4 \Lambda)^{1 / 2}},
$$




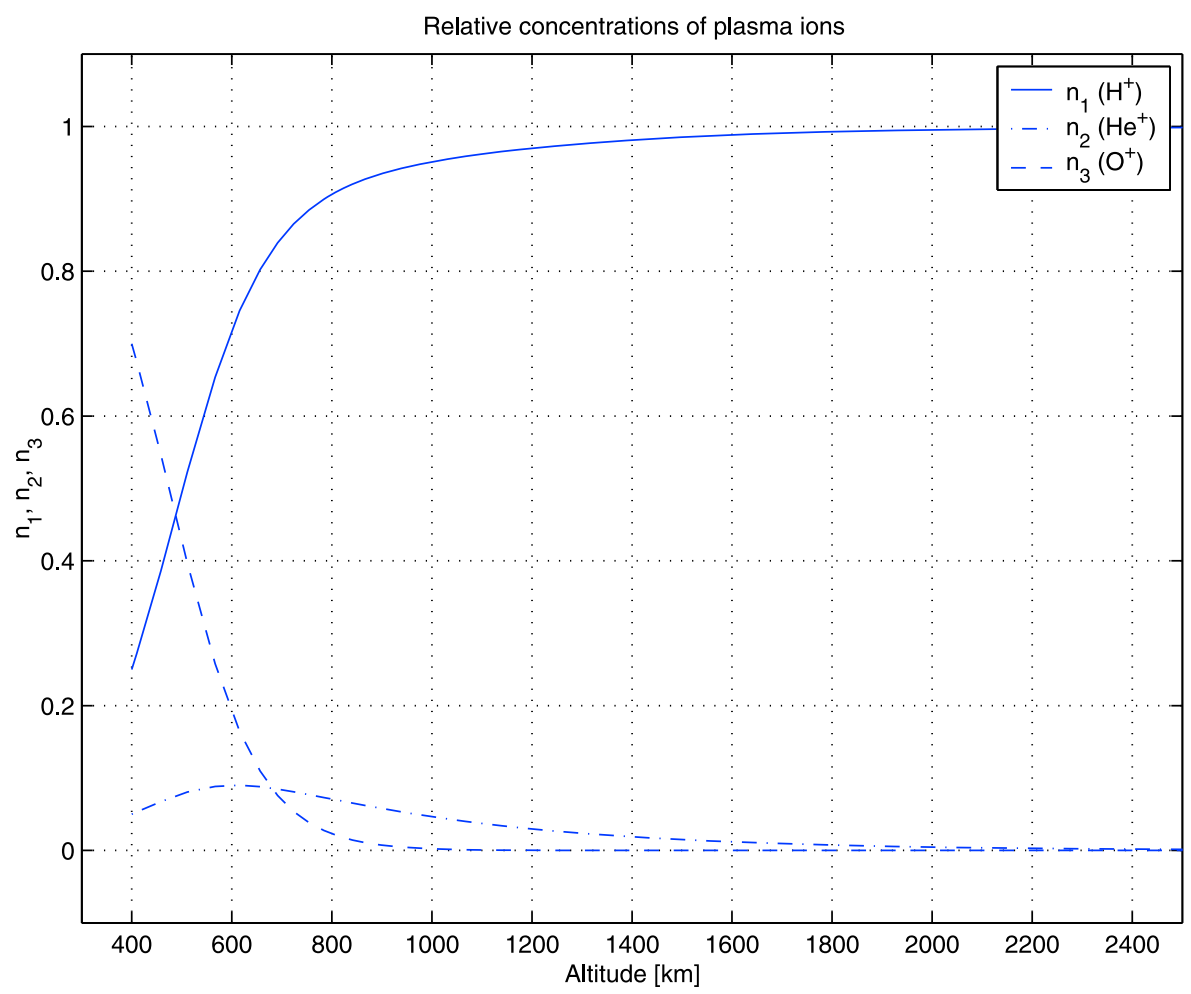

Figure A1. Relative concentrations of plasma ions as the functions of altitude used in ray tracing calculations.

and the quantity $\Lambda$ is expressed in terms of the geomagnetic latitude $\lambda$ as

$$
\Lambda=\operatorname{tg}^{2} \lambda \equiv \frac{Z^{2}}{X^{2}}
$$

In terms of these new variables, the equations of geometrical optics again have the Hamiltonian form, and they are:

$$
\begin{gathered}
\frac{d L}{d t}=-\frac{\partial F / \partial \kappa_{\mathrm{L}}}{\partial F / \partial \omega} ; \quad \frac{d M}{d t}=-\frac{\partial F / \partial \kappa_{\mathrm{M}}}{\partial F / \partial \omega} ; \\
\frac{d \kappa_{\mathrm{L}}}{d t}=\frac{\partial F / \partial L}{\partial F / \partial \omega} ; \quad \frac{d \kappa_{\mathrm{M}}}{d t}=\frac{\partial F / \partial M}{\partial F / \partial \omega} ;
\end{gathered}
$$

To define these equations completely, we need to specify models of the ambient magnetic field and of the plasma distribution. We use the centered dipole model for the geomagnetic field, which is reasonable for the upper ionosphere, and the gyrotropic model for the plasma density distribution: $\omega_{\mathrm{Pe}}^{2} \propto \omega_{\mathrm{He}}$. The ionic composition of the plasma is assumed to be altitude dependent, with the relative proportions of the different ions at the base level of $400 \mathrm{~km}$ as indicated in Figure 5, while the total ion density is equal to the electron density determined by the gyrotropic model. More precisely, the proton $(p)$, helium $\left(\mathrm{He}^{+}\right)$, and oxygen $\left(\mathrm{O}^{+}\right)$densities are taken, respectively, in the form:

$n_{p}(L, \lambda)=n_{1}(h) \cdot n_{e}(L, \lambda) ; n_{H e^{+}}(L, \lambda)=n_{2}(h) \cdot n_{e}(L, \lambda) ;$

$n_{O^{+}}(L, \lambda)=n_{3}(h) \cdot n_{e}(L, \lambda)$,

where electron density $n_{e}(L, \lambda)$ is written as

$$
n_{e}(L, \lambda)=\text { const } \cdot \omega_{\mathrm{He}} ; \quad \text { const }=0.0022 \mathrm{~s} / \mathrm{cm}^{3},
$$

and the dependences of $n_{1,2,3}$ on $h=R_{\mathrm{E}}[L /(1+\Lambda)-1]$ are shown in Figure A1. These last assumptions are serious simplifications, of course, but they should not lead to qualitative errors. A compelling argument in support of this statement is that the most important part of trajectory where $\omega \simeq \omega_{\mathrm{H} p}$ is correctly described by the model in use. The ray tracing results presented in section 3 are based on the solution of equations (A10) in the frame of the aforesaid assumptions.

[36] Acknowledgments. This work was supported by RFBR grants 10-02-93115-CNRS and 12-02-92614-RS. We also acknowledge support from grants 205/09/1253, 205/10/2279, and P209/12/P658 of the Grant Agency of the Czech Republic, and from the ME grant 09107. The authors thank J. J. Berthelier, the PI of electric field experiment on DEMETER, for providing the corresponding data. They also thank the reviewers for their helpful comments.

[37] Robert Lysak thanks the reviewers for their assistance in evaluating this paper.

\section{References}

Berthelier, J. J., et al. (2006), ICE, the electric field experiment on DEMETER, Planet. Space Sci., 54, 456-471, doi:10.1016/j.pss.2005.10.016.

Bošková, J., and F. Jiříček (1986), Deuteron whistlers in the outer ionosphere, Stud. Geophys. Geod., 30, 196-205.

Bošková, J., F. Jiříček, and P. Tř́ska (1976), Transequatorial propagation of ion-cyclotron waves, in Space Research XVI: Proceedings of the Open Meetings of Working Groups on Physical Sciences, pp. 435-439, AkademieVerlag GmbH, Berlin.

Bošková, J., F. Jiříček, and P. Tříska (1984), Deuteron whistlers at low latitudes in the low altitude topside ionosphere, ESA SP-217, pp. 543-547, ESA, Paris.

Carpenter, D. L., N. Dunckel, and J. F. Walkup (1964), A new very low frequency phenomenon: Whistlers trapped below the protonosphere, J. Geophys. Res., 69, 5009-5017, doi:10.1029/JZ069i023p05009.

Chum, J., F. Jiříček, and D. R. Shklyar (2003), Oblique noise bands above local LHR frequency, Adv. Space Res., 31, 1253-1258. 
Chum, J., O. Santolik, and M. Parrot (2009), Analysis of subprotonospheric whistlers observed by DEMETER: A case study, J. Geophys. Res., 114, A02307, doi:10.1029/2008JA013585.

Eckersley, T. L. (1935), Musical atmospherics, Nature, 135, 104-105.

Ferencz, C., O. Ferencz, D. Hamar, and J. Lichtenberger (2001), Whistler Phenomena: Short Impulse Propagation, Astrophy. Space Sci. Libr., vol. 262, Kluwer Acad., Dordrecht, Netherlands.

Ginzburg, V. L., and A. A. Rukhadze (1972), Waves in Magnetoactive Plasma, Handb. Phys., vol. 49, part IV, edited by S. Flügge, 395 pp., Springer, Berlin.

Gurnett, D. A., S. D. Shawhan, N. M. Brice, and R. L. Smith (1965), Ion cyclotron whistlers, J. Geophys. Res., 70(7), 1665-1688.

Helliwell, R. A. (1965), Whistlers and Related Ionospheric Phenomena, Stanford Univ. Press, Stanford, Calif.

Jones, D. (1969), The effect of the latitudinal variation of the terrestrial magnetic field strength on ion cyclotron whistlers, J. Atmos. Terr. Phys., 31, 971-981.

Kimura, I. (1966), Effects of ions on whistler-mode ray tracing, Radio Sci., 1, 269-283.

Likhter, Y. I., Y. P. Sobolev, F. Jiricek, and P. Triska (1974), Proton whistlers in the equatorial F-region, in Space Research XIV: Proceedings of the Open Meetings of Working Groups on Physical Sciences, pp. 265-269, Akademie-Verlag $\mathrm{GmbH}$, Berlin

Parrot, M. (Ed.) (2006), First results of the DEMETER micro-satellite, Planet. Space Sci., 54(5), 557 pp.

Parrot, M., et al. (2006), The magnetic field experiment IMSC and its data processing onboard DEMETER: Scientific objectives, description and first results, Planet. Space Sci., 54, 441-455, doi:10.1016/j.pss.2005.10.015.

Santolík, O., and M. Parrot (1999), Case studies on the wave propagation and polarization of ELF emissions observed by Freja around the local proton gyrofrequency, J. Geophys. Res., 104(A2), 2459-2475.

Santolík, O., F. Lefeuvre, M. Parrot, and J.-L. Rauch (2001), Complete wave-vector directions of electromagnetic emissions: Application to INTERBALL-2 measurements in the nightside auroral zone, J. Geophys. Res., 106, 13,191-13,201.

Santolík, O., J. S. Pickett, D. A. Gurnett, and L. R. O. Storey (2002), Magnetic component of narrow-band ion cyclotron waves in the auroral zone, J. Geophys. Res., 107(A12), 1444, doi:10.1029/2001JA000146.

Santolík, O., M. Parrot, and F. Lefeuvre (2003), Singular value decomposition methods for wave propagation analysis, Radio Sci., 38(1), 1010, doi:10.1029/2000RS002523.
Santolík, O., F. Němec, M. Parrot, D. Lagoutte, L. Madrias, and J. J. Berthelier (2006), Analysis methods for multi-component wave measurements on board the DEMETER spacecraft, Planet. Space Sci., 54, 512-527.

Shklyar, D. R. (2005), Key problems in numerical simulation of spectrograms related to emissions caused by lightning strokes, Geomagn. Aeron., $45,474-487$.

Shklyar, D. R., and F. Jiříček (2000), Simulation of nonducted whistler spectrograms observed aboard the MAGION 4 and 5 satellites, J. Atmos. Sol. Terr. Phys., 62, 347-370.

Shklyar, D. R., J. Chum, and F. Jiříček (2004), Characteristic properties of $\mathrm{Nu}$ whistlers as inferred from observations and numerical modelling, Ann. Geophys., 22, 3589-3606.

Shklyar, D. R., M. Parrot, J. Chum, O. Santolík, and E. E. Titova (2010), On the origin of lower- and upper-frequency cutoffs on wedge-like spectrograms observed by DEMETER in the midlatitude ionosphere, J. Geophys. Res., 115, A05203, doi:10.1029/2009JA014672.

Smith, R. L., and J. J. Angerami (1968), Magnetospheric properties deduced from OGO 1 observations of ducted and nonducted whistlers, J. Geophys. Res., 73, 1-20.

Smith, R. L., et al. (1964), An ion gyrofrequency phenomenon observed in satellites, Nature, 204(4955), 274-275.

Stix, T. H. (1992), Waves in Plasmas, Springer, New York.

Storey, L. R. O. (1953), An investigation of whistling atmospherics, Philos. Trans. R. Soc., 246, 113-141.

Storey, L. R. O., and F. Lefeuvre (1974), Theory for the interpretation of measurements of a random electromagnetic wave field in space, Space Res., 14, 381-386.

Storey, L. R. O., and F. Lefeuvre (1979), The analysis of 6-component measurement of a random electromagnetic wave field in a magnetoplasma: 1. The direct problem, Geophys. J. R. Astron. Soc., 56, 255-270.

Storey, L. R. O., and F. Lefeuvre (1980), The analysis of 6-component measurement of a random electromagnetic wave field in a magnetoplasma: 2. The integration kernels, Geophys. J. R. Astron. Soc., 62, 173-194.

Watanabe, S., and T. Ondoh (1976), Deuteron whistler and trans-equatorial propagation of the ion cyclotron whistler, Planet. Space Sci., 24(4), 359-364.

Watanabe, S., and T. Ondoh (1980), Propagation of trans-equatorial deuteron whistlers in the low latitude topside ionosphere, J. Atmos. Terr. Phys., 42, 427-435. 